\title{
Lesões Coronarianas de Bifurcação: Classificação, Estratégias de Tratamento e Perspectivas
}

\author{
Ricardo Pontes de Miranda ${ }^{1,2,3}$, Vinicius Monteiro Melo Filho ${ }^{1,2}$, Marcos de Oliveira Gusmão ${ }^{1,2}$, \\ Jorge Elysio Galvão Wanderley, ${ }^{1,2}$, Euler Guimarães Mesquita ${ }^{3}$, Nelson Fernando Eugenio Hurtado ${ }^{1}$
}

\section{RESUMO}

A ocorrência de obstruções coronarianas que envolvem ramos laterais, com maior ou menor risco de oclusão, não é um evento raro. O tratamento percutâneo destas lesões de bifurcação tem sido um desafio para a cardiologia intervencionista. A apresentação extremamente variada, seja pela disposição dos ramos, seja pelo comprometimento da placa sobre os ramos, propiciou a descrição de várias classificações. Ainda com a utilização de angioplastia coronária com cateter-balão, muitas técnicas foram descritas. Todas apresentavam resultados tardios pouco satisfatórios. Com o aparecimento dos stents na prática clínica, outras estratégias foram descritas, com a utilização de um e dois stents. No entanto, as taxas de revascularização da lesãoalvo permaneceram altas. O uso de stents com eluição de drogas, embora mostrando melhores resultados que os stents sem eluição, ainda não teve resultados absolutamente convincentes, especialmente em relação à ocorrência de restenose no óstio do ramo lateral. Apesar de não ser uma idéia nova, outros modelos de stents especialmente desenhados para o tratamento das lesões de bifurcação, chamados stents dedicados, estão sendo testados, alguns já comercializados. Os autores fazem uma revisão das classificações destas lesões e das estratégias de tratamento propostas, analisam as principais técnicas atualmente utilizadas, suas vantagens e desvantagens, e comentam os dispositivos em desenvolvimento para o tratamento destas obstruções coronárias.

DESCRITORES: Angioplastia transluminal percutânea coronária. Contenedores. Estenose coronária, terapia.

\footnotetext{
Clínica Cardiológica do Hospital Unimed Recife, PE.

${ }^{2}$ Setor de Cardiologia Intervencionista do Hospital Alfa - Recife, PE. ${ }^{3}$ Hospital Jayme da Fonte - Recife, PE.

Correspondência: Ricardo Pontes de Miranda. Rua Visconde de Jequitinhonha, 1144 - Sala 1202 - Boa Viagem - Recife - PE CEP 51030-020

E-mail: ricardopmiranda@hotmail.com

Recebido em: 8/2/2007 • Aceito em: 12/3/2007
}

\section{SUMMARY}

Coronary Bifurcation Lesions: Classification, Strategies of Treatment and Perspectives

The occurrence of coronary lesions that involve side branches, with greater or lesser risk of occlusion, is not a rare event. The percutaneous treatment of these bifurcation lesions is a challenge to interventional cardiology. Their extremely varied presentation, whether due to the arrangement of the branches, or to the plaque distribution in the branches, has resulted in the description of several classifications. When coronary angioplasty was performed only with balloon catheters, many techniques were described. Results at follow up were unsatisfactory. After the introduction of stents, other strategies, using one or two stents, were described. However, the rates of target lesion revascularization remained high. Although, the use of drug-eluting stents has shown better results than bare metal stents, they are not totally convincing yet, especially in regards to the occurrence of restenosis at the ostia of side branches. Despite of not being a new idea, other models of stents specially designed for the treatment of bifurcation lesions, called dedicated stents, are being tested and some are already on the market. The authors review the classifications of bifurcation lesions and of the proposed treatment strategies, analyze the main currently used techniques with their advantages and disadvantages, as well as comment on the devices being developed for the treatment of such coronary lesions.

DESCRIPTORS: Angioplasty, transluminal, percutaneous coronary. Stents. Coronary stenosis, therapy.

0 envolvimento de ramos laterais em obstruções coronárias não é um evento raro na cardiologia intervencionista. Cerca de metade das lesões coronarianas exibem ramo lateral em risco ${ }^{1}$.

Atualmente, devem merecer o termo lesão de bifurcação as lesões envolvendo ramos que tenham tamanho suficiente para serem tratados com o implante de stents. Deve-se considerar a bifurcação do tronco da coronária esquerda como um capítulo especial.

O tratamento destas lesões de bifurcação tem sido encarado, desde os primórdios da intervenção coronária 
percutânea, como um desafio para a cardiologia intervencionista. Isto se deve, sobretudo, à forma variada de apresentação, seja pelo ângulo formado por seus ramos, seja pela sua importância angiográfica, ou ainda, pelo grau de comprometimento dos mesmos.

Muitas classificações para este tipo de obstrução coronária foram descritas por diversos autores, sendo que, mais recentemente, propôs-se, em consenso, em reunião do European Bifurcation Club, a utilização de uma classificação única².

A escolha da técnica a ser utilizada para o tratamento deverá levar em conta a importância angiográfica e o tipo de comprometimento dos ramos, bem como o comportamento das placas após inflações com cateter-balão ou após o implante de stents.

Os avanços tecnológicos conseguidos, ao longo do tempo, na intervenção coronária percutânea, inclusive com a introdução dos stents com eluição de drogas (DES), vêm se traduzindo em um grande aumento do número de pacientes portadores deste tipo de obstrução tratados por via percutânea.

\section{CLASSIFICAÇÃO}

Habitualmente, para a classificação das lesões de bifurcação são levados em conta dois aspectos. O primeiro é relativo ao ângulo formado entre os ramos, dividindo as bifurcações nos grupos em forma de $Y$, se esse ângulo é menor que 70 graus e em forma de $\mathrm{T}$, se o ângulo é maior que este valor.

O outro aspecto é a distribuição da placa sobre os ramos. Algumas classificações foram propostas. As mais conhecidas são as de Lefèvre et al. ${ }^{3}$, Spokjony \& Sanborn ${ }^{4}$, Duke ${ }^{5}$, e Safian ${ }^{6}$. Recentemente, na cidade de Bordeaux, na França, em reunião do European Bifurcation Club, houve a recomendação para o uso da classificação de Medina et al. ${ }^{7}$ (Figura 1), que utiliza apenas a existência ou não de comprometimento maior que $50 \%$ de três pontos da bifurcação (as porções proximal e distal do ramo principal e o ramo lateral).

Em todas essas classificações, observa-se a presença das chamadas lesões verdadeiras de bifurcação (quando há envolvimento dos ramos principal e lateral de forma significativa) e as lesões falsas de bifurcação (onde há envolvimento significativo apenas do ramo principal ou lateral).

Um dos aspectos que envolvem a anatomia da bifurcação e da lesão é a redistribuição da placa, após insuflações com cateter-balão (pré-dilatação) ou com o implante de stents. Isso pode, eventualmente, forçar mudanças na estratégia de tratamento inicialmente programada.

A prática clínica tem mostrado que as bifurcações em forma de $\mathrm{T}$, embora apresentem maior dificuldade de acesso ao ramo lateral, têm menores índices de redistribuição da placa. O oposto é verdadeiro. As bifurcações em forma de $Y$ permitem acesso mais fácil ao ramo lateral, porém, apresentam mais freqüentes manifestações de redistribuição da placa. Aliabadi et al. ${ }^{8}$ estudaram lesões de bifurcação com ramos laterais maiores que $1 \mathrm{~mm}$ após o implante de stents liberados com alta pressão. Observaram oclusão total em 5,7\% dos ramos com lesão discreta (10/176) e 69\% dos ramos que apresentavam lesões acima de 50\% antes do procedimento (33/48).

Cuidadosa análise prévia pode ajudar na tentativa de se antecipar este comportamento. A realização da pré-dilatação é outro fator auxiliar nesta avaliação.

\section{HISTÓRICO}

Com a ampliação das indicações iniciais para angioplastia coronária, muitas técnicas foram descritas para o tratamento das lesões de bifurcação.

Ainda quando se utilizava apenas angioplastia coronária com cateter-balão, foram descritas técnicas que utilizaram dois cateteres-guia e inflações alternadas $^{9}$, a técnica kissing balloon (KB) utilizando dois cateteres-guia e inflações simultâneas ${ }^{10}$ e a mesma técnica KB utilizando um único cateter-guia e dois cateteres-balão de baixo perfil ${ }^{11}$.

Com o uso de stents de forma rotineira no tratamento das lesões coronárias, muitas outras técnicas foram descritas.

Colombo et al. ${ }^{12}$ descreveram o tratamento de dois pacientes utilizando a técnica kissing stent. Carrie et al. ${ }^{13}$ descreveram a técnica T-shaped com o uso de dois stents em forma de T. Outras estratégias como stent em $\mathrm{Y}^{14}$, stent em $\mathrm{V}^{15}$ e trouser-like ${ }^{16}$ foram descritas.

Chevalier et al. ${ }^{17}$ publicaram os resultados de uma

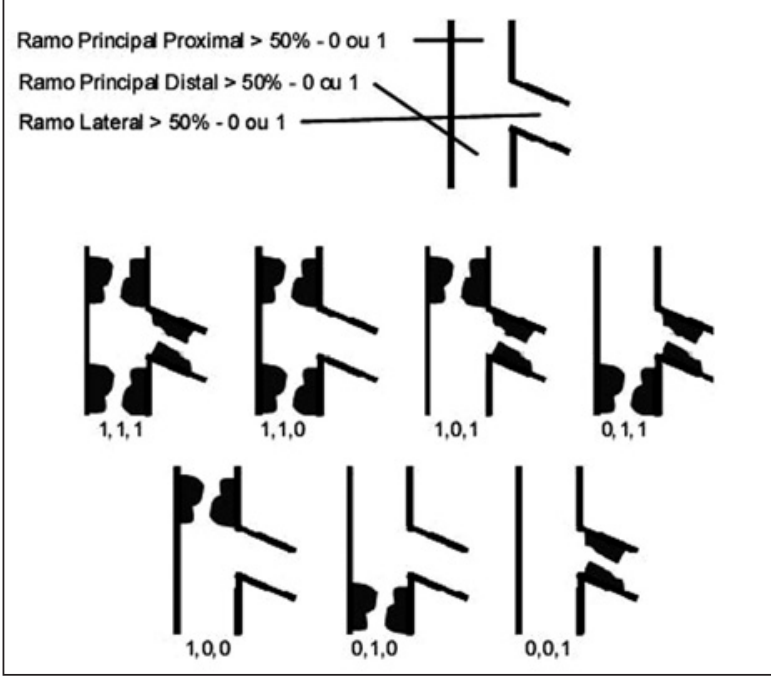

Figura 1 - Classificação de Medina et al. ${ }^{7}$. 
série de 50 pacientes nos quais utilizaram a técnica do culotte. O índice de sucesso primário foi de 94\% para colocação de dois stents. A taxa de revascularização da lesão-alvo (RLA) foi de $24 \%$. No seguimento angiográfico $(50 \%)$ foi identificada restenose em 14 pacientes. A não realização do KB após o procedimento foi associada a maior índice de nova intervenção (44\% versus $12 \%$ ).

Outros dispositivos foram usados para o tratamento das lesões de bifurcação. Eisenhauer et al. ${ }^{18}$ utilizaram aterectomia direcional (DCA). Karvouni et al. ${ }^{19}$ compararam aterectomia direcional seguida de implante de stent a apenas implante de stent. A taxa de RLA foi menor no grupo DCA comparado ao grupo stent, entretanto, o índice de eventos cardíacos adversos maiores (MACE) intra-hospitalar foi significativamente maior no grupo DCA (12,9\% versus $0 \%)$.

Embora todas estas estratégias tivessem a garantia de alto índice de sucesso, vinham acompanhadas de taxas elevadas de restenose, com conseqüente reintervenção.

A necessidade de se tornar o tratamento das lesões de bifurcação mais fácil e com melhores resultados estimulou o desenvolvimento de stents especialmente desenhados, chamados stents dedicados. Alguns modelos destes stents, como o Jomed Side-Branch ${ }^{\circledR}$ e Jomed Bifurcation ${ }^{\circledR}$, foram comercializados, inclusive no Brasil. O primeiro permitia acesso mais fácil ao ramo lateral, através de "janelas maiores", favorecendo o uso do stent provisional. O segundo, desenhado especialmente para a realização da técnica do culotte, também dispunha de desenho especial para facilitar o acesso ao ramo lateral. Ambos não eram pré-montados e isso fez com que sua utilização ficasse restrita, além disso, especialmente o Jomed Bifurcation ${ }^{\circledR}$, por utilizar, exclusivamente, a técnica do culotte, tornava o procedimento mais demorado e muito menos atrativo. Utilizamos o stent Jomed Bifurcation ${ }^{\circledR}$ em 44 bifurcações. Observamos taxas de RLA e MACE na evolução de sete meses de $22,7 \%$ e $31,8 \%$, respectivamente.

Com os bons resultados apresentados no uso dos stents com eluição de drogas, novas técnicas foram descritas para o tratamento das lesões de bifurcação. Colombo et al. ${ }^{20}$ descreveram a técnica do crushing, como uma alternativa à técnica $\mathrm{T}$.

Mais recentemente, novos stents dedicados têm sido comercializados; inicialmente em aço inoxidável, já começam a ser produzidos em cromo-cobalto. Por outro lado, diversos modelos de stents dedicados estão sendo desenvolvidos sem plataformas com eluição de drogas.

\section{AS TÉCNICAS}

A principal questão é a decisão pelo uso de um stent (stent no ramo principal e angioplastia com balão do ramo lateral) versus dois stents (uso provisional ou sistemático). As principais técnicas usadas são descritas a seguir.

\section{Técnica T e T Provisional}

A técnica em $\mathrm{T}$ tradicional (Figura 2) consiste em posicionar o primeiro stent no óstio do ramo lateral. Após sua liberação, recua-se o cateter-balão. O fio-guia do ramo lateral poderá ou não ser recuado. A seguir, o segundo stent é posicionado no ramo principal. Após a liberação do segundo stent, faz-se novo cruzamento do fio-guia para o ramo lateral e realiza-se o KB final.

O posicionamento do primeiro stent (ramo lateral) deve ser bastante cuidadoso, pois não deverá ficar com sua borda ainda no ramo principal. Isso eventualmente dificultará a progressão do segundo stent. Ademais, a liberação do stent do ramo principal, nesta eventualidade, poderá deformar a borda do stent do ramo lateral e dificultar o cruzamento do fio-guia e do cateter-balão para a realização do KB final. Recomenda-se, em algumas situações, o posicionamento do stent do ramo principal no local onde será liberado antes da liberação do stent do ramo lateral.

Por outro lado, em bifurcações em forma de $Y$, a liberação do stent no óstio do ramo lateral é ainda mais difícil que nas bifurcações em forma de T. Se o stent for posicionado muito avançado no ramo, deixará área sem cobertura metálica exatamente no óstio do ramo lateral. Este fato parece ser um dos preditores de restenose.

A técnica T provisional altera a ordem de liberação dos stents. Primeiro é liberado o stent do ramo principal. Em seguida, procede-se à passagem do fio-guia no ramo lateral, seguida de angioplastia com cateter-balão do seu óstio. De acordo com o resultado obtido faz-se a liberação do stent do ramo lateral, e, ao final, pósdilatação com KB. Nesta técnica, pode haver dificuldade de progressão do segundo stent (ramo lateral) através das estruturas do stent do ramo principal.

\section{Técnica V / Técnica Kissing Stent}

Estas técnicas têm como característica a liberação dos dois stents, de forma simultânea, nos ramos lateral e principal. Isto faz com que a carina da bifurcação seja refeita, imediatamente acima. Se essa nova carina se localiza numa distância menor que cinco milímetros da antiga, esta técnica é chamada de técnica $V$. Se for refeita numa distância maior, e, portanto, os stents se posicionam lado a lado na porção comum da bifurcação (ramo principal), ela é chamada de kissing stent (Figura 3).

Estas técnicas se aplicam melhor às bifurcações em forma de Y. Nas bifurcações em forma de T, o stent do ramo lateral pode não se adaptar bem ao ângulo, e a nova carina não ficar bem formada. Uma das grandes vantagens destas estratégias é que o acesso aos ramos principal e lateral não é perdido em nenhum momento do procedimento. Pode ser uma boa escolha nas lesões classificadas como $0,1,1$ ou mes- 


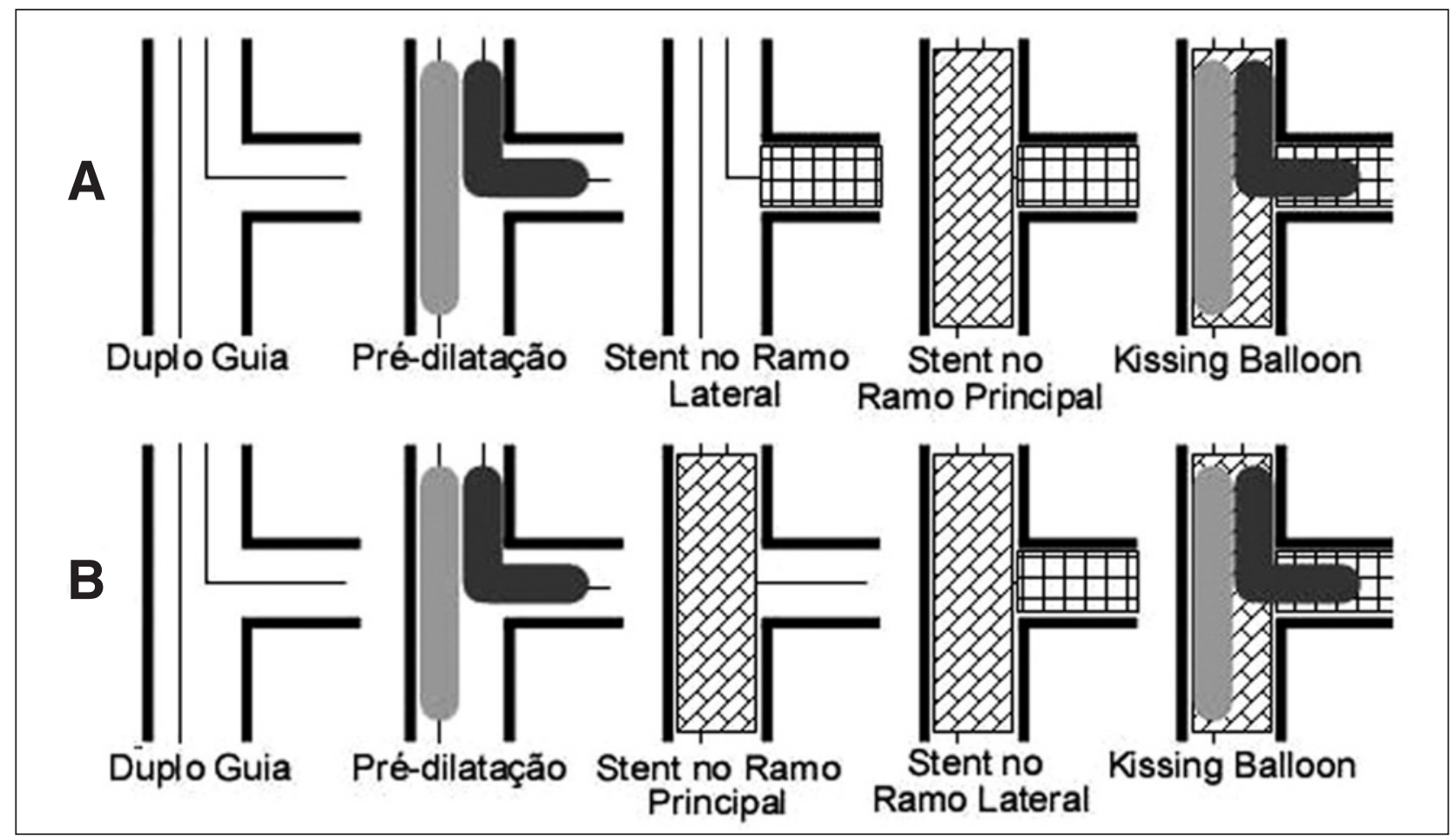

Figura 2 - A: Técnica T. B: Técnica T provisional.

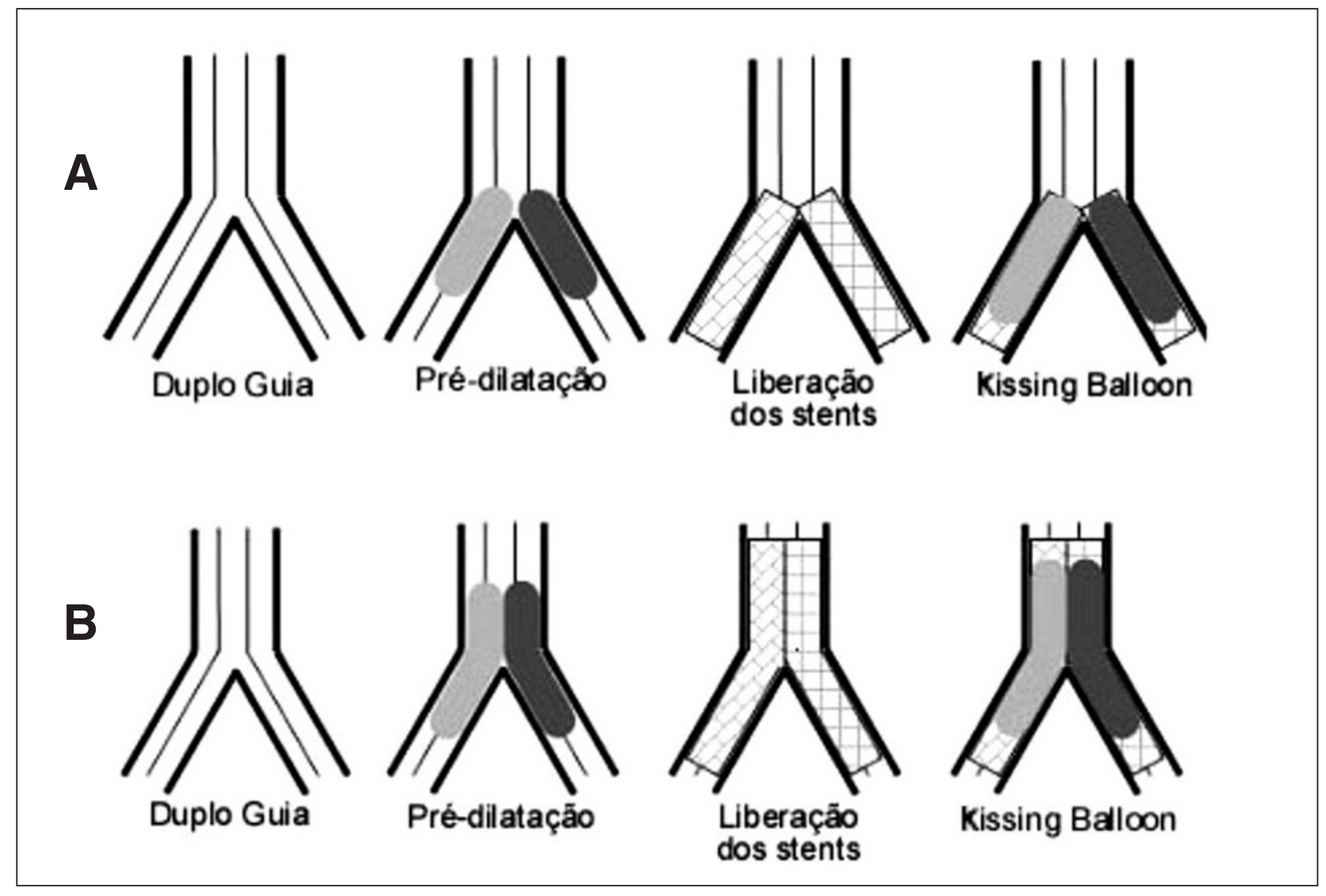

Figura 3 - A: Técnica V. B: Técnica kissing stent. 
mo nas lesões 1,1,1 (classificação de Medina) que tenham curto comprometimento da porção proximal do ramo principal.

\section{Técnica do Culotte}

A técnica do culotte utiliza sistematicamente dois stents (Figura 4). Após a pré-dilatação (isoladamente ou $K B)$, é liberado o primeiro stent. Alguns autores recomendam liberar no ramo mais angulado, normalmente o ramo lateral. Isso facilitaria o avanço do segundo stent. A seguir, recua-se o cateter-balão. Então se faz o cruzamento do segundo fio-guia para o outro ramo e liberase o segundo stent exatamente superposto ao primeiro, na porção proximal do ramo principal. Recua-se este balão e há a necessidade de novo cruzamento do primeiro fio-guia, para a realização do KB final.

A principal característica desta técnica é a completa cobertura metálica em todos os pontos da bifurcação. Entretanto, as principais críticas são a cobertura excessiva na porção proximal da bifurcação, onde há superposição dos stents, e a necessidade de duplo cruzamento dos fios-guia e dos cateteres-balão para a realização do KB final, especialmente se a pré-dilatação houver sido realizada com os dois fios-guia posicionados em seus respectivos ramos.

É evidente que o uso de stents superpostos na porção proximal da bifurcação resulta em cobertura metálica excessiva, e isto é um fator preditor para restenose. Em nossa experiência, utilizamos para esta técnica stents Jomed Bifurcation ${ }^{\circledR}$, que apresentavam um desenho especial com "células" maiores na porção proximal e, portanto, menor cobertura metálica individual e aceitável cobertura metálica quando superpostos. Porém, estes stents tiveram vida curta especialmente pelo fato de não serem comercializados na forma pré-montada.

\section{Técnica do Crushing}

A técnica do crushing foi descrita junto com a introdução dos stents com eluição de drogas, provavelmente pelos resultados iniciais bastante animadores com relação à restenose no uso destes dispositivos (Figura 4).

Ao utilizar dois stents sistematicamente, sua principal característica é a cobertura metálica de todos os pontos da bifurcação, à semelhança da técnica do culotte, porém com procedimento menos demorado e mais simples.

Inicia-se o procedimento com a liberação do stent do ramo lateral, deixando parte do stent ainda na porção proximal do ramo principal. A seguir, recua-se o cateter-balão e o segundo stent é liberado no ramo principal, "sepultando" contra a parede deste ramo parte do stent do ramo lateral. Após isso, é feito novo cruzamento do fio-guia do ramo lateral e realizado o $\mathrm{KB}$ final.

Se a técnica parece mais atrativa por assegurar a imediata patência dos ramos, em muitos casos não permite fácil cruzamento do fio-guia e do cateter-balão pelas estruturas dos stents, em função da deformação do stent do ramo lateral. Assim, em alguns casos, não se consegue realizar kissing balloon final.

Algumas variações desta técnica são o crush reverso e o step crush. Na primeira, o stent do ramo principal é liberado inicialmente. A seguir, é liberado o segundo stent (ramo lateral), de forma provisional, através das estruturas do stent do ramo principal, ficando parte ainda posicionada no ramo principal e com o cateter-balão do ramo principal já posicionado. O crush é realizado com a insuflação do cateter-balão do ramo principal e após novo cruzamento do fio-guia do ramo lateral, o KB finaliza o procedimento. Na segunda, em

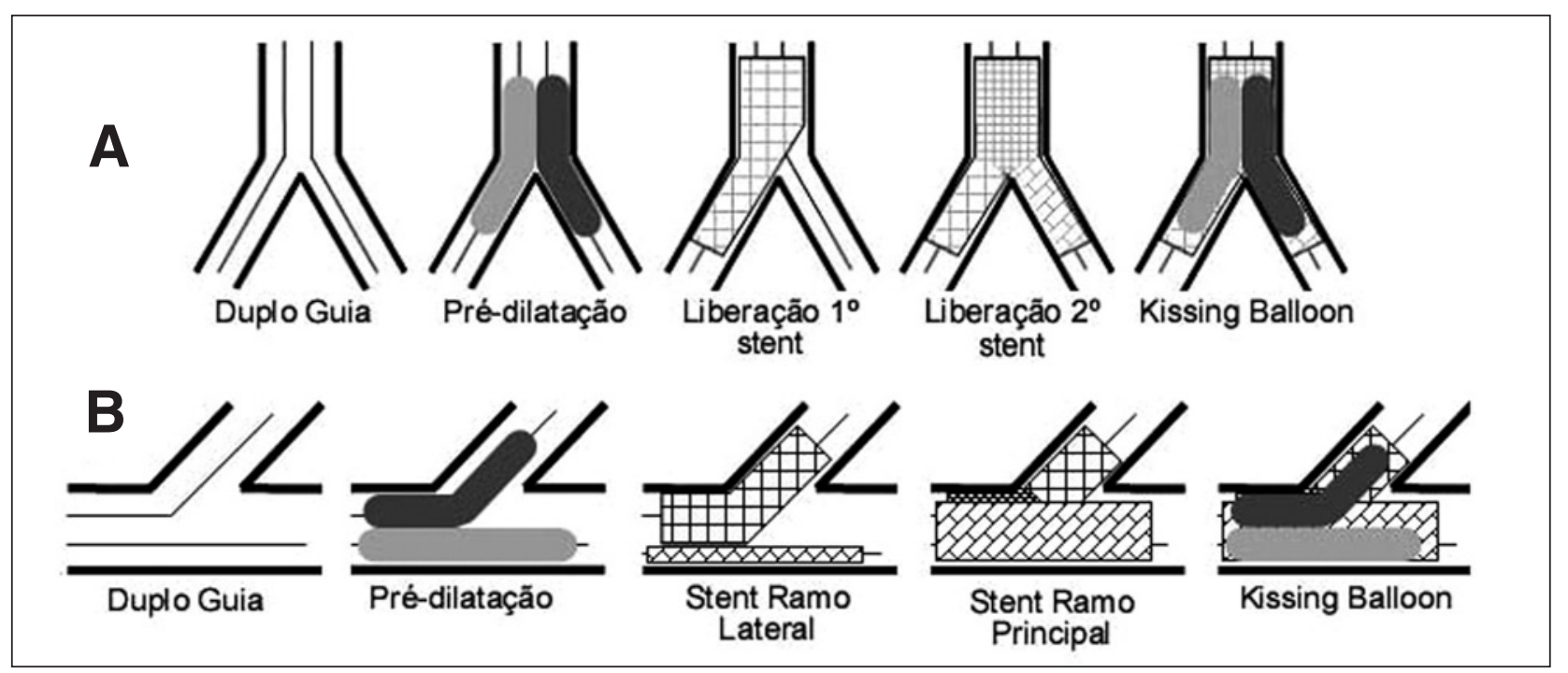

Figura 4 - A: Técnica do culotte. B: Técnica do crushing. 
uma adaptação para a utilização da técnica através de cateteres-guia $6 \mathrm{~F}$ e acesso radial, inicialmente é liberado o stent do ramo lateral, com um cateter-balão de baixo perfil posicionado no ramo principal. Recua-se o cateter-balão do ramo lateral e realiza-se o crush com a insuflação do cateter-balão no ramo principal. Retiram-se os cateteres-balão e faz-se a liberação do stent do ramo principal. Após novo cruzamento do fio-guia do ramo lateral, é feita a pós-dilatação com KB final utilizando-se cateteres-balões de baixo perfil.

\section{UM STENT VERSUS DOIS STENTS}

Parece claro que alguns fatores influenciam essencialmente a escolha da estratégia de tratamento percutâneo: 1- o ângulo entre os ramos da bifurcação, 2- a importância angiográfica do ramo lateral (diâmetro e extensão), 3- a forma de comprometimento da placa sobre os ramos, o que coloca o ramo lateral em maior ou menor risco de oclusão, 4- a gravidade do envolvimento ostial e 5- a filosofia e a experiência do operador.

O ângulo formado pelos ramos pode ser um fator determinante na escolha da estratégia de tratamento. Bifurcações que exibem ângulo entre os ramos acima de 70 graus podem significar maior dificuldade em avançar o stent provisional no ramo lateral. Já as bifurcações em forma de $Y$ normalmente oferecem menor dificuldade para o uso do segundo stent.

O tamanho do ramo lateral, assim como o risco de oclusão que a placa possa oferecer e a gravidade da lesão ostial determinam, em conjunto, a necessidade ou não de se "proteger" o ramo lateral, com o posicionamento de fio-guia, durante a pré-dilatação e liberação do stent do ramo principal.

Além disso, determinadas lesões "falsas" de bifurcação se transformam em lesões "verdadeiras", por redistribuição inesperada da placa. Isso também pode determinar a escolha final da estratégia a ser utilizada.

O desenho do stent não parece ser preditor de oclusão do ramo lateral. Cho et al. ${ }^{21}$, analisando um total de 322 lesões de bifurcação tratadas com três tipos de stent, não encontraram diferenças significativas no índice de oclusão dos ramos laterais. Primariamente, estes ocluíram quando apresentaram lesão ostial grave.

A principal questão é a decisão pelo uso de um stent (stent no ramo principal e angioplastia com balão do ramo lateral) versus dois stents (uso provisional ou sistemático). Yamashita et al. ${ }^{22}$ estudaram lesões de bifurcação tratadas com stent em ambos os ramos (grupo $B, n=53$ ), e com stent no ramo principal e angioplastia com balão do ramo lateral (grupo $\mathrm{P}, \mathrm{n}=39$ ). Menores índices de estenose residual foram evidenciados no grupo B $(7,4 \pm 10,9 \%$ vs. $23,4 \pm 18,7 \%)$. O índice de sucesso no procedimento foi semelhante nos dois grupos. A taxa de MACE na fase hospitalar ocorreu apenas no grupo B (13\% vs. 0\%). Na evolução de seis meses, a taxa de RLA foi similar (38\% vs. $36 \%$ ) e não houve diferença significativa nas taxas de MACE (51\% vs. 38\%).

Pan et al. ${ }^{23}$ compararam as mesmas técnicas. O sucesso do procedimento foi similar nos dois grupos (89\% versus 91\%). Entretanto, o índice de MACE aos 18 meses foi maior no grupo de dois stents (livre de eventos $44 \%$ vs. $75 \%$ ). Posteriormente, avaliaram outra série de 126 pacientes ${ }^{24}$, dividida em três grupos, numa estratégia "passo-a-passo". Inicialmente (passo 1) era implantado stent no ramo principal. A seguir, se necessário, realizada angioplastia com cateter-balão do ramo lateral através das estruturas do stent (passo 2). Finalmente, se o resultado não fosse satisfatório, utilizava-se o implante de stent no ramo lateral (passo 3). No primeiro passo, 35 $(28 \%)$ pacientes obtiveram sucesso no procedimento e 88 passaram ao passo 2. Nesta etapa, 76 (86\%) pacientes obtiveram sucesso no procedimento e cinco pacientes passaram ao passo seguinte (passo 3). Neste, todos os pacientes obtiveram sucesso no procedimento. O sucesso angiográfico nos dois ramos foi progressivamente maior (38\% vs. $88 \%$ vs. $92 \%)$. Entretanto, as taxas de RLA também foram progressivamente maiores $(13 \%$ vs. $16 \%$ vs. $20 \%$ ).

Estes e outros registros comparativos não foram avaliados pela "intenção de tratar". Muitos pacientes receberam stent no ramo lateral por causa de resultados não ideais. Todos têm conclusões muito parecidas em relação ao sucesso angiográfico primário, favoráveis ao grupo de dois stents. Entretanto, os índices de MACE, as taxas de RLA e restenose foram sempre maiores nos grupos de pacientes tratados com duplo stent, sendo esta última influenciada principalmente pelos índices de restenose do ramo lateral.

Entre as estratégias de duplo stent, a técnica do culotte parece ser a que oferece piores resultados, com altas taxas de MACE, restenose, trombose de stent e infarto do miocárdio ${ }^{26}$. A técnica $\mathrm{T}$ provisional mostrou melhores resultados que outras técnicas de duplo stent. Lefèvre et al. ${ }^{25}$ publicaram registro de quatro centros, que utilizou preferencialmente a técnica T provisional $(93,5 \%)$ e a técnica T $(3,7 \%)$. A evolução de seis meses mostrou índices de MACE de 16,0\% e RLA de 10,4\%.

Na nossa experiência, utilizando stents sem eluição de drogas, em pacientes com lesões de bifurcação que apresentavam ramo lateral com diâmetro igual ou maior que $2,5 \mathrm{~mm}, 38 \%$ dos pacientes (52/136) foram tratados com um stent no ramo principal e angioplastia com balão do ramo lateral e $62 \%(84 / 136)$ foram tratados por técnicas de duplo stent. Utilizamos as técnicas $T(n=24)$, culotte $(n=44)$ e $V(n=16)$. Na evolução de sete meses, a taxa de RLA no grupo com dois stents foi maior que no grupo de um stent $(21,4 \%$ vs. $15,4 \%)$. A taxa de MACE também foi maior no grupo de dois stents $(28,6 \%$ vs. 23,1\%). Individualmente, a técnica do culotte mostrou piores resultados em relação às técnicas $T$ e $V$, quanto à RLA e ao índice de MACE, mesmo utilizando stents especialmente desenhados para esta técnica. 
Louvard et al. ${ }^{26}$ propuseram uma nova classificação das estratégias de tratamento. Assim, o tratamento tipo A consiste no implante de stent a partir do óstio do ramo lateral, seguido de implante de stent no ramo principal "cobrindo" o óstio do ramo lateral. O tipo B consiste em liberação de stent no ramo principal, seguido por implante de stent a partir do óstio do ramo lateral através das estruturas do stent do ramo principal. Tipo C corresponde à técnica do culotte, conforme descrito anteriormente. O tipo D consiste na colocação de dois stents a partir do óstio dos ramos, seguida pela implantação de outro stent na porção proximal do ramo principal. Também chamada de técnica $Y$.

\section{STENTS COM ELUIÇÃO DE DROGAS}

Os resultados extremamente favoráveis inicialmente apresentados pelos stents com eluição de drogas estimularam os investigadores no tratamento das lesões de bifurcação. Os estudos iniciais com DES, comparados aos stents sem eluição de drogas em lesões não bifurcadas, mostraram grande diminuição das taxas de restenose mesmo com o uso de stents longos e superposição de stents. Assim, o uso de técnicas de duplo stent no tratamento deste tipo de lesão parecia o melhor caminho.

Com o objetivo de aferir a segurança e a viabilidade do uso de DES no tratamento de lesões de bifurcação, e de comparar o uso de um stent versus dois stents, Colombo et al. ${ }^{27}$ analisaram os resultados de uma série de pacientes, de diversos centros, tratados com stent com eluição de sirolimus. Quarenta e três pacientes foram inicialmente randomizados para o uso de dois stents e mesmo número de pacientes para o uso de stent no ramo principal e angioplastia com balão do ramo lateral. Vinte e dois pacientes que inicialmente foram designados para o uso de um stent necessitaram usar dois stents. A técnica $T$ foi utilizada em $95,2 \%$ dos pacientes. A trombose de stent ocorreu em três $(3,5 \%)$ pacientes, comprometendo o ramo lateral, em dois, e ambos os ramos, em um paciente. Um paciente teve morte súbita quatro meses após tratamento. Todos os pacientes que apresentaram trombose de stent e o paciente com morte súbita foram tratados com stent em ambos os ramos. Não houve diferença significativa na taxa de restenose no ramo principal entre o grupo de um stent comparado ao grupo de dois stents $(4,8 \%$ vs. 5,7\%). A taxa de restenose no ramo lateral foi mais alta no grupo de dois stents $(21,8 \%)$ quando comparada ao grupo de um stent $(14,1 \%)$, embora sem grande significado estatístico. A restenose do ramo lateral foi localizada principalmente no óstio e de aspecto focal (14/15 pacientes).

Os motivos pela ocorrência de alta taxa de restenose no óstio do ramo lateral não ficaram muito claros. Provavelmente, a dificuldade de posicionamento do stent no ramo lateral, impedindo uma completa aposição na carina, e sua deformação foram dois deles.
Por apresentar uma taxa de cross-over de cerca de $52 \%$, os resultados apresentados não puderam ser tomados como definitivos. No entanto, este estudo permitiu algumas conclusões iniciais: 1- o uso de DES em lesões de bifurcação resultou em baixas taxas de restenose no ramo principal, 2- a angioplastia com balão do óstio do ramo lateral freqüentemente apresentou resultado insatisfatório e obrigou o uso de stent, 3- o uso de stent no ramo lateral foi um preditor de mais altas taxas de restenose neste ramo, 4- o risco de trombose de stent foi maior que experiências anteriores com o uso de DES em situações menos complexas.

Mais recentemente, Ge et al. ${ }^{28}$ mostraram resultados semelhantes. Na evolução de nove meses, houve tendência a maiores índices de MACE e de RLA no grupo tratado com dois stents $(23,3 \%$ e $8,9 \%)$ comparado ao grupo tratado com um stent (18,9\% e 5,34\%), embora com pequeno significado estatístico.

Ao contrário do que se esperava, o uso da técnica do crushing não trouxe melhores resultados. Hoye et al. ${ }^{29}$ avaliaram os fatores preditores de resultados adversos em uma série de pacientes. Foram utilizados stents com eluição de sirolimus $(n=130)$ e de paclitaxel $(n=101)$. A sobrevida livre de RLA em nove meses foi de 90,3\% e o único fator preditor independente foi o implante de stent no tronco da coronária esquerda (TCE). A sobrevida livre de MACE foi $83,5 \%$ e os fatores preditores independentes de MACE foram terapia com stent do TCE e o tratamento de pacientes multiarteriais. A taxa de restenose binária foi de $9,1 \%$ e $25,3 \%$, para o ramo principal e ramo lateral, respectivamente. A perda tardia diminuiu significativamente no ramo lateral com a realização de KB final. Aos nove meses de evolução, $4,3 \%$ dos pacientes apresentaram um evento importante, com possível trombose de stent pós-procedimento. Os stents com sirolimus e com paclitaxel não mostraram diferenças significativas, incluindo aqueles que apresentaram restenose no ramo lateral.

Costa et al. ${ }^{30}$, usando ultra-som intravascular, avaliaram os resultados da técnica de crushing em 40 pacientes. Quando apenas o ramo principal foi avaliado, a menor área do stent localizava-se ao nível do crush em $56 \%$ dos casos comparada com a porção proximal e distal do stent. Quando ambos os stents foram considerados, a menor área foi localizada no óstio do ramo lateral em $68 \%$ dos pacientes. Crushing incompleto foi visto em $60 \%$ das bifurcações que não envolviam o tronco da coronária esquerda, e foi definido como a aposição incompleta das estruturas dos stents do ramo lateral ou ramo principal contra a parede do ramo principal proximal à carina. Este achado poderia ser o efeito da expansão do stent do ramo principal sobre o stent do ramo lateral, que "traciona" a porção do stent que deveria ficar aposta à carina. Isto pode contribuir para uma restenose mais alta nesta área.

Em publicação recente, Moussa et al. ${ }^{31}$ avaliaram 
a evolução clínica de pacientes tratados pela técnica do crushing com stents com eluição de sirolimus. Em seis meses, a taxa de RLA foi de 13,5\%. Destes, $69 \%$ apresentavam restenose no óstio do ramo lateral, com taxas de trombose subaguda de $1,7 \%$.

A restenose do óstio do ramo lateral tem sido o ponto comum a todos estes estudos, e é, neste momento, o "calcanhar-de-aquiles" em relação ao tratamento percutâneo das lesões de bifurcação. O uso de estratégias que permitam a cobertura metálica completa parecia ser uma possibilidade de solução. Assim, a técnica do culotte utilizando DES poderia ressurgir como uma grande alternativa.

Steigen et al. ${ }^{32}$ utilizaram stents com eluição de sirolimus no estudo Nordic e não encontraram diferenças significativas nas taxas de MACE entre os grupos de um stent e dois stents. O grupo que utilizou dois stents apresentou maior tempo de fluoroscopia, uso de maior quantidade de contraste e maior aumento de marcadores de injúria miocárdica. O objetivo angiográfico combinado, diâmetro da estenose no ramo principal maior que $50 \%$ e oclusão do ramo lateral após oito meses, não mostrou diferença significativa entre os dois grupos (5,3\% no grupo um stent e 5,1\% no grupo dois stents). Concluíram que os resultados clínicos e angiográficos obtidos foram excelentes, independente da técnica utilizada.

\section{STENTS DEDICADOS}

A idéia de se utilizar dispositivos que tornassem mais fácil o tratamento percutâneo de lesões de bifurcação não é nova.

Mais recentemente, novos projetos estão sendo desenvolvidos e alguns já comercializados. Os modelos mais conhecidos são stents montados sobre dois balões, sendo que parte de um deles encontra-se dirigida por uma das "janelas" do stent para o ramo lateral, facilitando a progressão deste balão.

Com algumas diferenças nas suas propostas, têm como característica comum a garantia do acesso ao ramo lateral, seja para a realização de angioplastia com balão, seja para o uso do stent provisional. Alguns modelos oferecem também, pelo seu desenho, a cobertura completa do óstio do ramo lateral. Assim, utilizase a técnica T provisional, e a decisão do implante do segundo stent dependerá do resultado obtido após a angioplastia do ramo lateral. Para a sua utilização há a necessidade de se posicionar os fios-guia no ramo principal e lateral, antes do avanço do sistema.

Utilizando o stent Multi-Link Frontier ${ }^{\circledR}$, Lefèvre et al. ${ }^{33}$ avaliaram os resultados de registro multicêntrico. O sucesso do procedimento foi observado em 93\% dos pacientes. Na evolução aos 30 dias e seis meses, a taxa de MACE foi de $2,9 \%$ e $17,1 \%$ (nenhuma morte ou trombose subaguda), respectivamente. A restenose intra-stent e "no segmento" do ramo principal foi de $25,3 \%$ e $29,9 \%$, respectivamente. A restenose do ramo lateral foi de $29,1 \%$.

Por serem montados sobre dois balões, têm um perfil mais alto e menor flexibilidade. Isto pode dificultar a navegabilidade do sistema, especialmente em artérias coronárias tortuosas. A evolução destes dispositivos para modelos em cromo-cobalto, com perfis menores, poderá melhorar sua flexibilidade.

Por outro lado, os fios-guia ao serem posicionados no início do procedimento podem se cruzar várias vezes ao longo do cateter-guia, e impedir o avanço do stent, obrigando o recuo de um deles, seguido de novo avanço, para que se consiga o posicionamento ideal.

Existem poucos dados referentes aos stents dedicados. Resultados preliminares de pequenas séries têm revelado segurança no uso, baixas taxas de trombose e resultados relativamente animadores em relação à restenose e aos índices de MACE.

Nossa experiência com o uso destes sistemas, ainda pequena, apresenta resultados semelhantes aos dados até agora publicados. A proposta de facilitação do acesso ao ramo lateral e também de melhor cobertura do seu óstio nos parece promissora. Por enquanto, estes stents estão disponíveis com uma única opção de comprimento e algumas opções de diâmetro. Em situações de lesões mais extensas no ramo principal, existe a necessidade do uso de um ou mais stents adicionais.

Diversos stents dedicados em plataformas com eluição de drogas estão sendo desenvolvidos, seja para uso provisional T (Twin-Rail ${ }^{\circledR}, \mathrm{Nile}^{\circledR}$, Frontier $^{\circledR}$, Petal $\left.{ }^{\circledR}\right)$, stents dedicados para ramo lateral $\left(\right.$ Tryton $^{\circledR}$, Capella ${ }^{\circledR}$ ) e stents dedicados à carina $\left(\right.$ Axxess $\left.{ }^{\circledR}\right)$.

Existe a necessidade de maior quantidade de estudos para uma melhor avaliação do desempenho. Acreditamos que estes stents não serão usados na prática clínica antes de provarem ter resultados semeIhantes aos stents tubulares quanto ao sucesso angiográfico, duração do procedimento e conseqüente exposição radiológica, taxa de complicações agudas e índices de MACE e RLA ${ }^{26}$.

\section{ALGUMAS OBSERVAÇÕES PRÁTICAS}

O tratamento percutâneo das lesões de bifurcação começa na avaliação prévia da lesão. Assim, lesões que possuem ramo lateral em risco, freqüentemente, necessitam do uso de dois stents.

Por isso, há a necessidade de se projetar o comportamento da lesão, após a pré-dilatação ou após o implante do stent do ramo principal, para que se possa antecipar a estratégia final a ser utilizada.

Conseqüentemente, a escolha do cateter-guia de- 
penderá da estratégia escolhida e do modelo de stent(s) a ser(em) utilizado(s).

Ao utilizar stents sem eluição de drogas, pode-se usar os cateteres-guia $7 \mathrm{~F}$ com diâmetro interno, no mínimo, 0,078 polegadas, em praticamente todas as técnicas, inclusive naquelas em que os stents são liberados simultaneamente.

Para os stents dedicados, utilizamos rotineiramente os cateteres-guia 7F (diâmetro interno 0,076 polegadas, no mínimo), embora os fabricantes assegurem um perfil do stent de 0,070 polegadas, possibilitando o uso em cateteres-guia 6F com diâmetro interno 0,071 polegadas.

Para as técnicas de liberação simultânea de stents (técnica $\mathrm{V}$ ou kissing stent) e a técnica do crushing, utilizamos cateteres-guias com diâmetro interno 0,087 polegadas (8F). Para as técnicas T e T provisional, embora possam ser utilizados cateteres-guia $6 \mathrm{~F}$, na nossa prática diária, preferimos utilizar cateteres-guia 7F.

Sistematicamente deve ser utilizada a pré-dilatação. Entretanto, é importante evitar a ocorrência de dissecção na região da bifurcação. Isso poderia dificultar o acesso posterior. Escolher os cateteres-balão de acordo com os diâmetros dos ramos pode minimizar esta ocorrência. Pode ser feita isoladamente ou com KB, embora não haja vantagens evidentes desta última.

Ao posicionar os dois fios-guia nos ramos principal e lateral no início do procedimento, deve-se evitar manusear em excesso o segundo guia para diminuir o cruzamento entre eles. Ao utilizar os stents dedicados e houver cruzamento dos fios-guia, recuar sempre o do ramo lateral no sistema $\mathrm{Nile}^{\circledR}$ e qualquer um no sistema Twin-Rail ${ }^{\circledast}$.

A permanência do fio-guia do ramo lateral, durante a liberação do stent do ramo principal, traz algumas vantagens. A modificação de angulação da bifurcação com a manutenção do fio-guia facilita a passagem através das estruturas do stent. Pode também manter fluxo coronário nos casos de oclusão do ramo lateral após a liberação do stent do ramo principal. A ruptura do fio-guia é rara.

Entretanto, é necessário cuidado na liberação do stent do ramo principal. Se este stent é liberado com diâmetro maior que o diâmetro do vaso, pode haver resistência ao recuo do fio-guia e o conseqüente avanço do cateter-guia, com risco de dano à porção proximal da artéria coronária. Assim, deve-se liberar o stent numa relação 0,9:1 ou 1:1 em relação ao diâmetro do vaso.

Finalmente, quando se realiza angioplastia com balão do ramo lateral, isoladamente, pelas estruturas do stent do ramo principal, há deformação na parede contra-lateral do stent. Por isso, utilizar sempre o KB final em todas as técnicas de duplo stent, como também na técnica de stent no ramo principal com angioplastia com balão do ramo lateral.

\section{CONCLUSÕES}

A ocorrência de lesões de bifurcação não é rara e seu tratamento percutâneo permanece um desafio para a cardiologia intervencionista.

Apesar dos avanços conseguidos nesta área, ainda não há uma estratégia de tratamento preferencial. O uso dos stents com eluição de drogas não apresentou resultados absolutamente satisfatórios. Os stents dedicados podem ser um bom caminho a ser seguido.

Entretanto, há a necessidade de mais dados para que conclusões definitivas possam ser feitas. A forma extremamente variada de apresentação das lesões de bifurcação, assim como a inexistência de padrão para a redistribuição da placa, dificultam a realização de estudos randomizados.

A análise cuidadosa da lesão a ser tratada ajudará na escolha da estratégia e no modelo de stent.

À luz dos dados até agora apresentados, parece claro que, com o uso de stents sem eluição de drogas, a estratégia $T$ provisional é superior às que fazem uso sistemático de duplo stent. Com o emprego de stents com eluição de drogas, os dados ainda são insuficientes.

As taxas de restenose, especialmente no ramo lateral, parecem estar intimamente relacionadas à cobertura ideal e geometricamente uniforme de seu óstio. Os principais objetivos a serem alcançados são dispositivos mais simples de serem usados e que apresentem resultados absolutamente idênticos aos obtidos no tratamento das lesões não bifurcadas.

\section{REFERÊNCIAS BIBLIOGRÁFICAS}

1. Meier B, Gruentzig AR, King SB, Douglas JS, Hollman J, Ischinger $\mathrm{T}$, et al. Risk of side branch occlusion during coronary angioplasty. Am J Cardiol. 1984;53(1):10-4.

2. Thomas M, Hildick-Smith D, Louvard Y, Albiero R, Darremont O, Stankovic G, et al. Percutaneous coronary intervention for bifurcation disease. A consensus view from the first meeting of European Bifurcation Club. Eurointerv. 2006;2:149-53.

3. Lefèvre $T$, Louvard $Y$, Morice MC, Dumas P, Loubeyre C, Benslimane A, et al. Stenting of bifurcation lesions: classification, treatments, and results. Catheter Cardiovasc Interv. 2000;49(3):274-83.

4. Spokjony AM, Sanborn TM. The Bifurcation lesion. In: Ellis SG, Holmes DR Jr, eds. Strategic approaches in coronary intervention. Baltimore: Williams e Wilkins; 1996. p.288.

5. Popma JJ, Leon MB, Topol EJ. Atlas of interventional cardiology. Philadelphia: Saunders; 1994.

6. Safian RD. Bifurcation lesions. In: Safian RD, Freed MS, eds. The manual of interventional cardiology. Royal Oak: Physician's Press; 2001. p.222.

7. Medina A, Suárez de Lezo J, Pan M. A new classification of coronary bifurcation lesions. Rev Esp Cardiol. 2006;59(2):183.

8. Aliabadi D, Tilli FV, Bowers TR, Benzuly KH, Safian RD, Goldstein JA, et al. Incidence and angiographic predictors of side branch occlusion following high-pressure intracoronary stenting. Am J Cardiol. 1997;80(8):994-7. 
9. Zack PM, Ischinger T. Experience with a technique for coronary angioplasty of bifurcational lesions. Cathet Cardiovasc Diagn. 1984;10(5):433-43.

10. George BS, Myler RK, Stertzer SH, Clark DA, Cote G, Shaw $R E$, et al. Balloon angioplasty of coronary bifurcation lesions: the kissing balloon technique. Cathet Cardiovasc Diagn. 1986;12(2):124-38.

11. Thomas ES, Williams DO. Simultaneous double balloon coronary angioplasty through a single guiding catheter for bifurcation lesions. Cathet Cardiovasc Diagn. 1988;15(4):260-4.

12. Colombo A, Gaglione A, Nakamura S, Finci L. "Kissing" stents for bifurcational coronary lesion. Cathet Cardiovasc Diagn. 1993;30(4):327-30.

13. Carrie D, Karouny E, Chouairi S, Puel J. " $\mathrm{T}$ "-shaped stent placement: a technique for the treatment of dissected bifurcation lesions. Cathet Cardiovasc Diagn. 1996;37(3):311-3.

14. Fort S, Lazzam C, Schwartz L. Coronary "Y" stenting: a technique for angioplasty of bifurcation stenoses. Can J Cardiol. 1996;12(7):678-82.

15. Schampaert E, Fort S, Adelman AG, Schwartz L. The VStent: a novel technique for coronary bifurcation stenting. Cathet Cardiovasc Diagn. 1996;39(3):320-6.

16. Khoja A, Ozbek C, Bay W, Heisel A. Trouser-like stenting: a new technique for bifurcation lesions. Cathet Cardiovasc Diagn. 1997;41(2):192-6.

17. Chevalier B, Glatt B, Royer T, Guyon P. Placement of coronary stents in bifurcation lesions by the "culotte" technique. Am J Cardiol. 1998;82(8):943-9.

18. Eisenhauer AC, Clugston RA, Ruiz CE. Sequential diretional atherectomy of coronary bifurcation lesions. Cathet Cardiovasc Diagn. 1993;Suppl 1:54-60.

19. Karvouni E, Di Mario C, Nishida T, Tzifos V, Reimers B, Albiero $\mathrm{R}$, et al. Directional atherectomy prior to stenting in bifurcation lesions: a matched comparison study with stenting alone. Catheter Cardiovasc Interv. 2001;53(1):12-20.

20. Colombo A, Stankovic G, Orlic D, Corvaja N, Liistro F, Airoldi F, et al. Modified T-stenting technique with crushing for bifurcations lesions: immediate results and 30-day outcome. Catheter Cardiovasc Interv. 2003;60(2):145-51.

21. Cho GY, Lee CW, Hong MK, Kim JJ, Park SW, Park SJ. Effects of stent design on side branch occlusion after coronary stent placement. Catheter Cardiovasc Interv. 2001;52(1):18-23.

22. Yamashita $T$, Nishida $T$, Adamian MG, Briguori $C$, Vaghetti $\mathrm{M}$, Corvaja $\mathrm{N}$, et al. Bifurcation lesions: two stents versus one stent-immediate and follow-up results. J Am Coll Cardiol. 2000;35(5):1145-51.

23. Pan M, Suárez de Lezo J, Medina A, Romero M, Hernández E, Segura J, et al. Simple and complex stent strategies for bifurcated coronary arterial stenosis involving the side branch origin. Am J Cardiol. 1999;83(9):1320-5.

24. Pan M, Suárez de Lezo J, Medina A, Romero M, Segura J, Ramírez A, et al. A stepwise strategy for the stent treatment of bifurcated coronary lesions. Catheter Cardiovasc Interv. 2002;55(1):50-7.

25. Lefèvre T, Guyon P, Brunel L, Maillard L, Louvard Y, Glatt $B$, et al. Stenting of bifurcation lesions using the BX Velocity Stent - a multicenter French Study. Arch Mal Coeur. 2002;95(Suppl III):513.

26. Louvard Y, Lefèvre T, Morice MC. Percutaneous coronary intervention for bifurcation coronary disease. Heart. 2004;90 (6):713-22.

27. Colombo A, Moses JW, Morice MC, Ludwig J, Holmes DR, Spanos V, et al. Randomized study to evaluate sirolimuseluting stents implanted at coronary bifurcation lesions. Circulation. 2004;109(10):1244-9.

28. Ge L, Tsagalou E, lakovou I, Sangiorgi GM, Corvaja N, Airoldi $F$, et al. In-hospital and nine-month outcome of treatment of coronary bifurcational lesions with sirolimuseluting stent. Am J Cardiol. 2005;95(6):757-60.

29. Hoye A, lakovou I, Ge L, van Mieghem CA, Ong AT, Cosgrave J, et al. Long-term outcomes after stenting of bifurcation lesions with the "crush" technique: predictors of an adverse outcome. J Am Coll Cardiol. 2006;47(10):1949-58.

30. Costa RA, Mintz GS, Carlier SG, Lansky AJ, Moussa I, Fujii $\mathrm{K}$, et al. Bifurcation coronary lesions treated with the "crush" technique: an intravascular ultrasound analysis. J Am Coll Cardiol. 2005;46(4):599-605.

31. Moussa I, Costa RA, Leon MB, Lansky AJ, Lasic Z, Cristea $\mathrm{E}$, et al. A prospective registry to evaluate sirolimus-eluting stents implanted at coronary bifurcation lesions using the "crush technique". Am J Cardiol. 2006;97(9):1317-21.

32. Steigen TK, Maeng M, Wiseth R, Erglis A, Kumsars I, Narbute I, et al. Randomized study on simple versus complex stenting of coronary artery bifurcation lesions: the Nordic bifurcation study. Circulation. 2006;114(18):1955-61.

33. Lefèvre T, Ormiston J, Guagliumi G, Schultheiss HP, Quilliet L, Reimers B, et al. The Frontier stent registry: safety and feasibility of a novel dedicated stent for the treatment of bifurcation coronary artery lesions. J Am Coll Cardiol. 2005;46(4):592-8. 